Research Paper

\title{
AIBI Genomic Amplification Predicts Poor Clinical Outcomes in Female Glioma Patients
}

\author{
Lihong Chen ${ }^{*}$, Changwei Wang ${ }^{2}$, Xinyuan Zhang 3 , Ke Gao ${ }^{4}$, Rui Liu ${ }^{5}$, Bingyin Shi ${ }^{6,7}$, and Peng Hou ${ }^{6,7 凶}$ \\ 1. Department of Critical Care Medicine, The First Affiliated Hospital of Xi'an Jiaotong University, Xi'an 710061, The People's Republic of China \\ 2. Guangzhou Institute of Biomedicine and Health, Chinese Academy of Sciences, Guangzhou 510530, The People's Republic of China \\ 3. Department of Biochemistry, McMaster University, Hamilton, Ontario L8S 4L8, Canada \\ 4. Department of Neurosurgery, The First Affiliated Hospital of Xi' an Jiaotong University, Xi' an 710061, The People's Republic of China \\ 5. Department of Radio Oncology, The First Affiliated Hospital of Xi' an Jiaotong University, Xi'an 710061, The People's Republic of China \\ 6. Department of Endocrinology, The First Affiliated Hospital of Xi'an Jiaotong University, Xi'an 710061, The People's Republic of China \\ 7. Key Laboratory for Tumor Precision Medicine of Shaanxi Province, The First Affiliated Hospital of Xi'an Jiaotong University, Xi' an 710061, The People's \\ Republic of China. \\ * These authors contributed equally to this work.
}

$\triangle$ Corresponding author: Peng Hou, Ph.D., Key Laboratory for Tumor Precision Medicine of Shaanxi Province, The First Affiliated Hospital of Xi'an Jiaotong University, Xi' an 710061, The People's Republic of China. Tel/Fax: +86 298532 3259; Email: phou@xjtu.edu.cn

(C) Ivyspring International Publisher. Reproduction is permitted for personal, noncommercial use, provided that the article is in whole, unmodified, and properly cited. See http://ivyspring.com/terms for terms and conditions.

Received: 2016.05.05; Accepted: 2016.08.14; Published: 2016.10.22

\begin{abstract}
Amplified in breast cancer I (AIBI) gene, a coactivator for steroid receptor, is frequently amplified in diverse cancers and is considered as an oncogene in tumorigenesis. However, the prognostic significance of $A I B I$ amplification in gliomas remains totally unclear. In this study, 115 gliomas and 16 benign meningiomas as control subjects were enrolled, and the copy number of AIBI was analyzed in these samples. In addition, we explored potential correlation of $A I B I$ amplification with clinicopathological characteristics and clinical outcomes of glioma patients. Our data showed that glioma samples exhibited a significantly higher AIBI copy number than control subjects as determined by quantitative polymerase chain reaction ( $\mathrm{PPCR}$ ) approach. Moreover, univariate analysis showed that AIBI amplification ( $\geq 3.5$ copies) was strongly correlated with cancer-related death $(P=0.03)$. Interestingly, our data revealed a significant association of $A I B I$ amplification with WHO grade $(P=0.03)$, tumor recurrence $(P=0.03)$ and survival status $(P=0.03)$ in female patients but not in male patients. Multivariate analysis further demonstrated that $A I B I$ amplification was independent factor for cancer-related death in female patients. Importantly, $A I B I$ amplification was closely relevant to worse survival in female patients $(P=0.001)$, but not in male patients $(P=1.00)$. In addition, the patients with AIBI amplification were resistant to radiotherapy. Altogether, our data demonstrate that $A I B I$ amplification is a common genetic event in glioma tumorigenesis, and suggest that $A I B I$ amplification is not only a prognostic factor for poor clinical outcomes in glioma patients, but also a predictor of radiotherapy resistance in gliomas.
\end{abstract}

Key words: Glioma, AIB1 amplification, clinical outcomes, radiotherapy resistance

\section{Introduction}

Glioma is the most prevalent primary brain tumor in the central nervous system (CNS) and is characterized by high recurrence and mortality rates $[1,2]$. Gliomas are generally categorized into four grades (I-IV) based on the World Health Organization (WHO) classification, including pilocytic astrocytoma (PA), diffuse astrocytoma (DA), anaplastic astrocytoma (AA) and glioblastoma (GBM) [3]. Despite considerable progresses in the application of comprehensive treatment strategies for glioma patients, the 5-year survival rate still remains poor [4, 5]. Therefore, valuable genomic biomarkers with increased sensitivity and reliability are critically required for predicting clinical outcomes and 
establishing new therapeutic and preventive strategies for gliomas.

Gene amplification is considered as a pivotal molecular process of tumorigenesis through increasing gene copy number and subsequently activating the oncogenic potential of proto-oncogenes [6]. Similar to other tumors, glioma is characterized by changes in the expression of oncogenes and tumor suppressor genes due to numerical chromosomal abnormalities such as genomic gains and losses [7-10]. Molecular profiling of glioblastoma has recently demonstrated that expression of $\sim 76 \%$ of genes with recurrent genomic copy number alterations (CNAs) is closely correlated with their copy number [7].

AIB1 (also known as steroid receptor coactivator-3, SRC-3) is a member of the p160 steroid receptor coactivator family. It is well-known that AIB1 plays an oncogenic function in tumorigenesis by affecting several important carcinogenic signaling pathways [11, 12]. In recent years, AIB1 has been reported to be frequently amplified in different types of cancer such as breast, ovarian, esophageal, colorectal, hepatocellular, gastric, pancreatic, bladder, nasopharyngeal and non-small-cell lung cancers (NSCLC) [12-20], and this genetic event is correlated with poor prognosis, aggressive tumor phenotype, progression and metastasis of tumors [21-23]. A recent study has revealed that AIB1 protein levels are much higher in high-grade astrocytomas than that in low-grade astrocytomas [24]. However, the prognostic significances of AIB1 genomic amplification in gliomas remain totally unclear.

In this study, AIB1 copy number was investigated in a cohort of gliomas and control subjects using qPCR approach. In addition, the correlation between AIB1 amplification and clinical outcomes of glioma patients was also explored in this study.

\section{Methods}

\section{Patients and Tissue Samples}

A total of 115 glioma patients and 16 benign meningiomas as control subjects (10 females and 6 males, age $54.6 \pm 7.1$ years), who underwent surgery for brain tumors at the Department of Neurosurgery of First Affiliated Hospital of Xi'an Jiaotong University from 2006 to 2012, were randomly enrolled in this study. All patients did not receive radiotherapy or chemotherapy prior to surgery. Glioma patients received adjuvant radiotherapy and/or chemotherapy after surgery according to standard clinical protocols. All samples were histopathologically classified according to the WHO classification criteria. Overall survival was calculated as time duration starting from surgery until cancer-related death or last follow-up. Clinicopathological data of patients were presented in Table 1. All of the patients were enrolled after providing a written informed consent. This study was approved by the institutional review board.

Table 1. Clinicopathological characteristics of glioma patients.

\begin{tabular}{ll}
\hline Characteristics & No. of patients (\%) \\
\hline Gender & $64(55.7)$ \\
Male & $51(44.3)$ \\
Female & \\
Age, years & 45.3 \\
Mean & 16.5 \\
Standard deviation & \\
WHO grade & $16(13.9)$ \\
I & $57(49.6)$ \\
II & $28(24.3)$ \\
III & $14(12.2)$ \\
IV & \\
Recurrence & $86(74.8)$ \\
Yes & $29(25.2)$ \\
No & \\
Radiotherapy & $72(62.6)$ \\
Yes & $43(37.4)$ \\
No & \\
Chemotherapy & $48(41.7)$ \\
Yes & $67(58.3)$ \\
No & \\
KPS sore & $45(39.1)$ \\
High & $70(60.9)$ \\
Low & \\
Epilepsy & $54(47.0)$ \\
Yes & $61(53.0)$ \\
No & $33(28.7)$ \\
Smoking & $82(71.3)$ \\
Yes & \\
No & $66(57.4)$ \\
Survival status & $49(42.6)$ \\
Dead &
\end{tabular}

\section{Tissues and DNA Preparation}

Formalin-fixed paraffin-embedded tissues were cut at $5 \mathrm{~mm}$, and stained by hematoxylin and eosin (H\&E). Tumor representative tissues were marked in each section by an expert cancer pathologist. Manual microdissection was carried out under an inverted light microscope by using the marked sections. DNA was extracted according to a previously described protocol [25]. In brief, the sections were first treated with xylene for $12 \mathrm{~h}$ at room temperature for deparaffinization, followed by digestion with $1 \%$ sodium dodecylsulfate (SDS) and proteinase $\mathrm{K}$ at $48^{\circ} \mathrm{C}$ for $48 \mathrm{~h}$. Genomic DNA was then isolated from these tissues using a standard protocol. 
Table 2. The primer and TaqMan probe sequences used in this study.

Gene $\quad$ Forward primer sequence $\left(5^{\prime} \rightarrow 3^{\prime}\right) \quad$ Probe sequence $\left(5^{\prime} \rightarrow 3^{\prime}\right)$

AIB1 CCTTACCAGGGTGAATTTTTATTG 6FAM-ATCTGTGTGGCACGCCGCATTACTACA-TAMRA

Reverse Primer sequence $\left(5^{\prime} \rightarrow 3^{\prime}\right)$

$\begin{array}{lll}\text { AIB1 } & \text { CCTTACCAGGGTGAATTTTTTATTG } & \text { 6FAM-ATCTGTGTGGCACGCCGCATTACTACA-T } \\ \beta \text {-actin } & \text { TCACCCACACTGTGCCCATCTACGA } & \text { 6FAM-ATGCCCTCCCCCATGCCATCC-TAMRA }\end{array}$

GGGTTTGATGGAAATGTTCTTTCT

\section{Copy Number Analysis}

Copy number of AIB1 was analyzed in gliomas and control subjects by a well-established real-time quantitative PCR approach, which was previously validated by fluorescence in situ hybridization (FISH) [26, 27]. Primer Express 3.0 software (Applied Biosystems, Foster City, CA) was utilized to design specific PCR primers and TagMan probes for the amplification of AIB1 gene and internal control $\beta$-actin. TaqMan probes were labeled at the 5 ' end with a fluorescent reporter 6-carboxyfluorescein (6FAM) and at the $3^{\prime}$ end with a fluorescent quencher 6-carboxy-tetramethylrhodamine (TAMRA). The sequences were presented in Table 2. The PCR reaction was performed according to a previously described protocol [26]. Each sample was run in triplicate, and $\beta$-actin was performed in parallel to normalize the input DNA. Serially diluted leukocyte DNA was used to establish standard curves. DNA copy number was calculated as previously described $[27,28]$. A copy number $\geq 3.5$ was defined as gene amplification (or copy gain).

\section{Statistical Analysis}

Statistical analysis was performed using the SPSS 11.5 software (Chicago, IL, USA). $P$ value $<0.05$ was considered statistically significant. Mann-Whitney $U$-test was applied to compare the copy number of AIB1 between gliomas and control subjects. SPSS 11.5 software was used to univariately analyze the correlation of AIB1 copy number and clinicopathological features. Multivariate analysis was performed to calculate multivariable-adjusted odds ratios (ORs) and 95\% confidence intervals (CIs) for AIB1 copy number, and other factors such as age, recurrence, radiotherapy and epilepsy. Cancer-related survival was calculated from the date of the operation to cancer-related death or last follow-up. Kaplan-Meier survival analysis was performed to evaluate the effect of AIB1 amplification on patient survival. Log-rank test was used to analyze the differences between curves. The impact of AIB1 amplification on the independent survival of age, radiotherapy and WHO grade was determined by multivariate Cox regression analysis.

\section{Results}

\section{Frequent AIBI Amplification in Gliomas}

The copy number of AIB1gene was examined in a cohort of gliomas and control subjects using qPCR assay. As shown in Figure 1, glioma patients exhibited significantly higher copy number of AIB1 than the controls (meningioma patients) (Median, 2.78 copies vs. 1.99 copies; $P=0.0003$ ). When a copy number of $\geq 3.5$ was considered as gene amplification, we found AIB1 amplification in 28/115 (24.3\%) gliomas, whereas none in control subjects. To test the relationship between of AIB1 copy number and its mRNA expression, we analyzed the corresponding data in a total of 435 low-grade gliomas using The Cancer Genome Atlas (TCGA) dataset from the Cancer Browser database (https://genome-cancer. soe.ucsc.edu). We divided all cases into low copy (L)-, median copy (M)- and high copy $(\mathrm{H})$-groups by use of two cutoff points (the 25 and 75 percentile of AIB1 copy number). As shown in Figure 2A, mRNA expression of AIB1 in H-group was significantly higher than that $\mathrm{M}$ - and L-groups. Given that chemotherapy and radiation therapy may affect mRNA expression of AIB1, we only analyzed their association in the cases who did not receive any therapy. Similar to the findings in Figure 2A, we still found a high mRNA expression of AIB1 in H-group compared with M- and L-groups (Figure 2B).

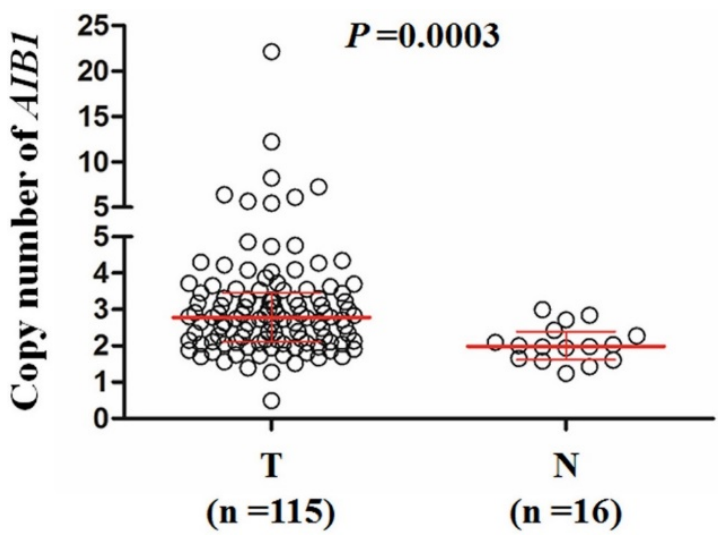

Figure 1. Copy number of $A I B I$ in a cohort of gliomas and control subjects. $A I B I$ copy number of each case was determined by a qPCR assay. Each circle represents the AIBI copy number of an individual case. Horizontal lines indicate median and inter-quartiles (25-75\%). T: tumor tissues; $\mathrm{N}$ : control subjects. 
A

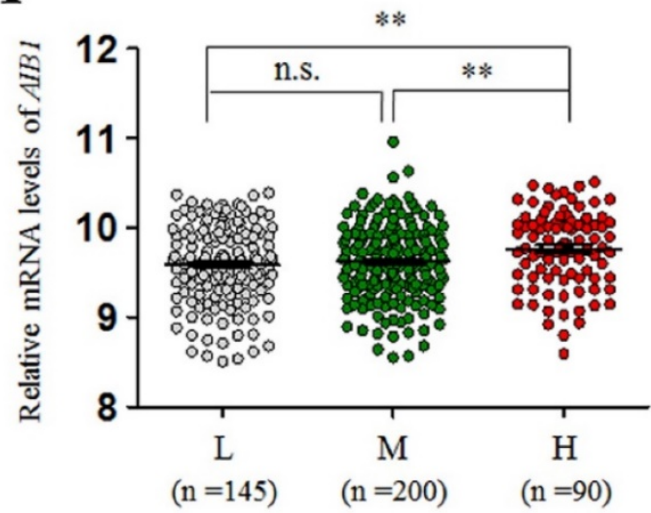

B

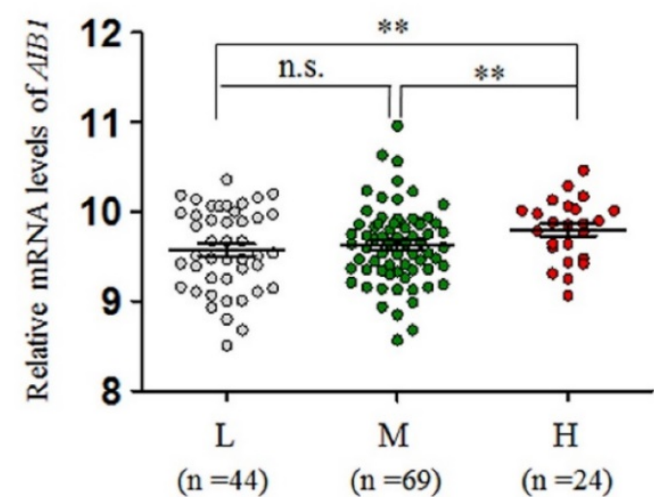

Figure 2. The relationship between copy number of AlB land its mRNA expression in low-grade gliomas from The Cancer Genome Atlas (TCGA) dataset. (A) All glioma patients. (B) The patients who did not receive chemotherapy or radiation therapy. Horizontal lines indicate median and inter-quartiles (25-75\%). L, low copy number of $A I B I ; M$, medium copy number of $A I B I ; H$, high copy number of $A I B I ; * *, P<0.01$.

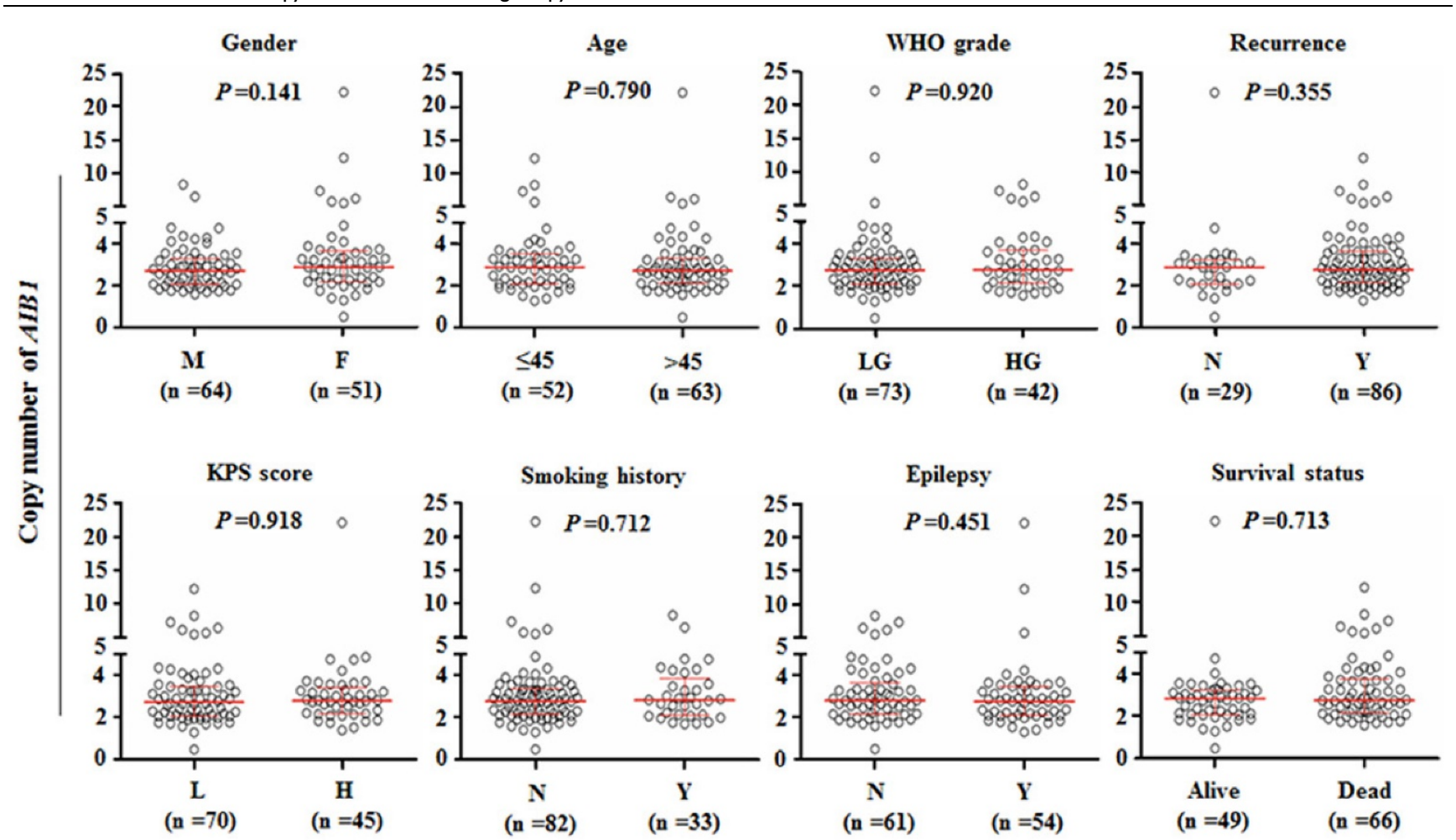

Figure 3. Relationship between AIBI copy number and clinicopathological features in glioma patients. Copy number of $A I B I$ was evaluated by a qPCR method. Each circle represents the AIBI copy number of an individual case. Horizontal lines indicate median and inter-quartiles (25-75\%). Mann-Whitney $U$ test was used for the comparison of sample medians. M: male; F: female; LG: low-grade tumors; HG: high-grade tumors; L: low; H: high; N: No; Y: Yes.

Next, we analyzed the AIB1 copy number grouped by the indicated clinicopathological features such as gender, age, WHO grade, tumor recurrence, Karnofsky performance status (KPS) score, smoking history, epilepsy and survival status. As shown in Figure 3, our data did not show significant relationships between AIB1 copy number and clinicopathological features. However, we noted that copy number of AIB1was slightly higher in female patients than male patients (Median, 2.87 copies vs. 2.69 copies).

\section{Association of AIBICopy Number with Clinicopathological Features in Gliomas}

Given frequent AIB1 amplification in gliomas, the relationships between AIB1 amplification and clinicopathological features were investigated in a cohort of gliomas. We defined a copy number of $\geq 3.5$ as amplification. The glioma patients were subsequently categorized into AIB1 amplification and non-amplification groups. As shown in Table 3, the risk of cancer-related death was significantly increased by the presence of AIB1 amplification (OR 
$=2.80,95 \% \mathrm{CI}=1.08-7.26 ; P=0.03)$. However, there were no significant correlations between AIB1 amplifications and other clinicopathological characteristics such as gender, age, WHO grade, recurrence, radiotherapy, chemotherapy, KPS score, epilepsy and smoking.

Table 3. AIBI amplification in gliomas: univariate associations with clinicopathological characteristics.

\begin{tabular}{|c|c|c|}
\hline \multirow[t]{2}{*}{ Characteristics } & \multicolumn{2}{|l|}{ Copy number } \\
\hline & $\mathrm{OR}^{*}(95 \% \mathrm{CI})$ & $P$ \\
\hline Gender & $0.61(0.26-1.44)$ & 0.26 \\
\hline Age $^{1}$ & $0.78(0.33-1.82)$ & 0.56 \\
\hline WHO grade 2 & $1.23(0.75-2.00)$ & 0.41 \\
\hline Recurrence & $2.42(0.76-7.69)$ & 0.13 \\
\hline Radiotherapy & $2.12(0.81-5.51)$ & 0.12 \\
\hline Chemotherapy & $0.87(0.37-2.09)$ & 0.76 \\
\hline KPS score ${ }^{3}$ & 0.99 (0.41-2.37) & 0.99 \\
\hline Epilepsy & $0.80(0.34-1.90)$ & 0.62 \\
\hline Smoking & $1.24(0.50-3.13)$ & 0.64 \\
\hline Survival status 4 & $2.80(1.08-7.26)$ & 0.03 \\
\hline
\end{tabular}

${ }^{*}$ OR: odds ratio with $95 \%$ confidence interval $(\mathrm{CI}) ;{ }^{1}$ Age (per 10 years); ${ }^{2} \mathrm{WHO}$ grade (I, II, III and IV); ${ }^{3} \mathrm{KPS}$ (>80; $\left.\leq 80\right) ;{ }^{4}$ Survival status (alive $v$ s. dead).

The patients were further categorized into two groups according to gender. Interestingly, we did not find that AIB1 amplification was correlated with cancer-related death $(\mathrm{OR}=0.95,95 \% \mathrm{CI}=0.27-3.33 ; P$ $=0.94$ ) in male patients (Table 4). However, we found a significant association of AIB1 amplification with cancer-related death $(\mathrm{OR}=11.50,95 \% \mathrm{CI}=2.24-59.01$; $P=0.03$ ) in female patients (Table 4). Moreover, AIB1 amplification was significantly associated with WHO grade $(\mathrm{OR}=4.00,95 \% \mathrm{CI}=1.11-14.43 ; P=0.03)$ and tumor recurrence $(\mathrm{OR}=11.20,95 \% \mathrm{CI}=1.33-94.49 ; \mathrm{P}$ $=0.03$ ) in female patients (Table 4). Next, multiple multivariable logistic regressions were conducted to analyze the independent association of AIB1 amplification with age, WHO grade, radiotherapy, epilepsy and cancer-related death. Similar to the findings from univariate analysis, AIB1 amplification was still closely correlated with cancer-related death in glioma patients $(\mathrm{OR}=3.76,95 \% \mathrm{CI}=1.21-11.66$; $P$ $=0.02)$, particularly in female patients $(\mathrm{OR}=10.60,95 \%$ $\mathrm{CI}=1.56-72.14 ; P=0.02)$ (Table 5).

\section{Effect of AIBIAmplification on Poor Survival of Glioma Patients}

We next conducted the univariate survival analysis to determine the potential relationship between AIB1 amplification and poor patient survival. As shown in Table 6, AIB1 amplification was notably correlated with poor survival of patients $(\mathrm{HR}=1.77$, $95 \% \mathrm{CI}=1.05-2.98 ; P=0.03)$. Further analysis showed a significant relationship between AIB1 amplification and poor survival in female patients ( $\mathrm{HR}=3.41,95 \%$ $\mathrm{CI}=1.57-7.43 ; P=0.002$ ), but not in male patients (HR $=1.00,95 \% \mathrm{CI}=0.46-2.18 ; P=0.99)$. In order to clarify clinical significance of AIB1 amplification in prognosing patient survival, Cox multivariate regression analysis was conducted in the present study. Also shown in Table 6, AIB1 amplification was identified as an independent variable for predicting the poor survival in glioma patients $(\mathrm{HR}=1.78,95 \%$ $\mathrm{CI}=1.00-3.13 ; P=0.048$ ).

Table 4. AIBI amplification in female and male glioma patients: univariate associations with clinicopathological characteristics.

\begin{tabular}{lllll}
\hline \multirow{2}{*}{ Characteristics } & \multicolumn{3}{c}{ Female patients } & Male patients \\
\cline { 2 - 5 } & OR* $^{*}(\mathbf{9 5} \% \mathbf{C I})$ & $\mathbf{P}$ & OR $^{*}(\mathbf{9 5} \% \mathbf{C I})$ & $\mathbf{P}$ \\
\hline Age $^{1}$ & $1.02(0.31-3.42)$ & 0.97 & $0.60(0.18-2.04)$ & 0.41 \\
WHO grade $^{2}$ & $4.00(1.11-14.43)$ & 0.03 & $0.82(0.43-1.57)$ & 0.55 \\
Recurrence $_{\text {Radiotherapy }}$ & $11.20(1.33-94.49)$ & 0.03 & $0.71(0.16-3.13)$ & 0.66 \\
Chemotherapy & $1.67(0.72-18.88)$ & 0.12 & $1.31(0.38-4.57)$ & 0.67 \\
KPS score $^{3}$ & $1.50(0.46-5.25)$ & 0.48 & $0.50(0.14-1.83)$ & 0.30 \\
Epilepsy $_{\text {Survival status }}{ }^{4}$ & $0.78(0.23-2.62)$ & 0.69 & $0.76(0.22-2.65)$ & 0.67 \\
\hline
\end{tabular}

${ }^{*}$ OR: odds ratio with $95 \%$ confidence interval (CI); ${ }^{1}$ Age (per 10 years); ${ }^{2} \mathrm{WHO}$ grade (I, II, III and IV); ${ }^{3} \mathrm{KPS}(>80 ; \leq 80) ;{ }^{4}$ Survival status (alive $v$ s. dead).

Table 5. AIBI amplification in all and female glioma patients: multivariable models assessing age, WHO grade, radiotherapy, epilepsy and survival status.

\begin{tabular}{|c|c|c|c|c|c|c|}
\hline \multirow[t]{2}{*}{ Characteristics } & \multicolumn{2}{|l|}{ All patients } & \multicolumn{2}{|c|}{ Female patients } & \multicolumn{2}{|c|}{ Male patients } \\
\hline & $\begin{array}{l}\mathrm{OR}^{*}(\mathbf{9 5 \%} \\
\mathrm{CI})\end{array}$ & $\mathbf{P}$ & $\begin{array}{l}\mathrm{OR}^{*}(95 \% \\
\mathrm{CI})\end{array}$ & $\mathbf{P}$ & $\begin{array}{l}\text { OR* }(95 \% \\
\text { CI) }\end{array}$ & $\mathbf{P}$ \\
\hline Age $^{1}$ & $\begin{array}{l}0.65 \\
(0.25-1.68)\end{array}$ & 0.38 & $\begin{array}{l}1.20 \\
(0.24-5.97)\end{array}$ & 0.82 & $\begin{array}{l}0.58 \\
(0.15-2.22)\end{array}$ & 0.42 \\
\hline WHO grade ${ }^{2}$ & $\begin{array}{l}0.98 \\
(0.55-1.74)\end{array}$ & 0.95 & $\begin{array}{l}1.06 \\
(0.38-3.00)\end{array}$ & 0.91 & $\begin{array}{l}0.78 \\
(0.36-1.67)\end{array}$ & 0.51 \\
\hline Radiotherapy & $\begin{array}{l}2.59 \\
(0.94-7.15)\end{array}$ & 0.07 & $\begin{array}{l}3.64 \\
(0.51-25.86)\end{array}$ & 0.20 & $\begin{array}{l}1.22 \\
(0.30-5.00)\end{array}$ & 0.78 \\
\hline Epilepsy & $\begin{array}{l}0.93 \\
(0.36-2.40)\end{array}$ & 0.87 & $\begin{array}{l}1.09 \\
(0.23-5.14)\end{array}$ & 0.91 & $\begin{array}{l}0.62 \\
(0.15-2.54)\end{array}$ & 0.50 \\
\hline $\begin{array}{l}\text { Survival } \\
\text { status }^{3}\end{array}$ & $\begin{array}{l}3.76 \\
(1.21-11.66)\end{array}$ & 0.02 & $\begin{array}{l}10.60 \\
(1.56-72.14)\end{array}$ & 0.02 & $\begin{array}{l}1.35 \\
(0.26-6.98)\end{array}$ & 0.72 \\
\hline
\end{tabular}

${ }^{*}$ OR: odds ratio with $95 \%$ confidence interval $(\mathrm{CI}) ;{ }^{1}$ Age (per 10 years); ${ }^{2} \mathrm{WHO}$ grade (I, II, III and IV); ${ }^{3}$ Survival status (alive $v$ s. dead).

Table 6. Prognostic value of clinicopathological factors and AIBI amplification using univariate and multivariate Cox regression analysis $(n=115)$.

\begin{tabular}{|c|c|c|c|c|}
\hline \multirow[t]{2}{*}{ Variables } & \multicolumn{2}{|c|}{ Univariate analysis } & \multicolumn{2}{|l|}{ Multivariate analysis } \\
\hline & $\begin{array}{l}\text { Hazard Ratio } \\
(95 \% \mathrm{CI})\end{array}$ & $P$ & $\begin{array}{l}\text { Hazard Ratio (95\% } \\
\text { CI) }\end{array}$ & $P$ \\
\hline \multicolumn{5}{|c|}{ Copy number } \\
\hline$<3.50$ & 1.00 (reference) & & 1.00 (reference) & \\
\hline$\geq 3.50$ & $1.77(1.05-2.98)$ & 0.03 & $1.78(1.00-3.13)$ & 0.048 \\
\hline \multicolumn{5}{|l|}{ Age } \\
\hline$\leq 45$ & 1.00 (reference) & & 1.00 (reference) & \\
\hline$>45$ & $2.26(1.36-3.76)$ & 0.002 & $2.05(1.21-3.46)$ & 0.008 \\
\hline \multicolumn{5}{|c|}{ Radiotherapy } \\
\hline No & 1.00 (reference) & & 1.00 (reference) & \\
\hline Yes & $0.51(0.31-0.82)$ & 0.006 & $0.51(0.30-0.85)$ & 0.01 \\
\hline \multicolumn{5}{|c|}{ WHO grade } \\
\hline I & 1.00 (reference) & & 1.00 (reference) & \\
\hline II & $2.43(0.83-7.13)$ & 0.11 & $2.51(0.84-7.49)$ & 0.10 \\
\hline III & $9.93(3.34-29.52)$ & $<0.001$ & $7.66(2.52-23.31)$ & $<0.001$ \\
\hline IV & $\begin{array}{l}10.10 \\
(3.17-32.25)\end{array}$ & $<0.001$ & $10.12(3.10-33.04)$ & $<0.001$ \\
\hline
\end{tabular}


A

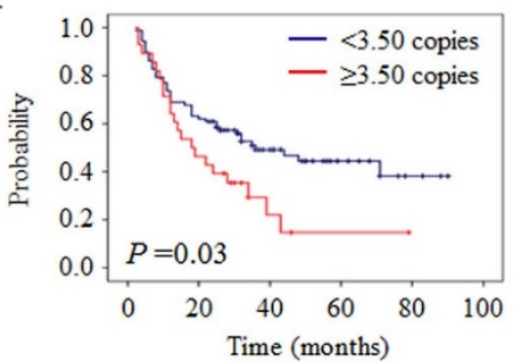

B

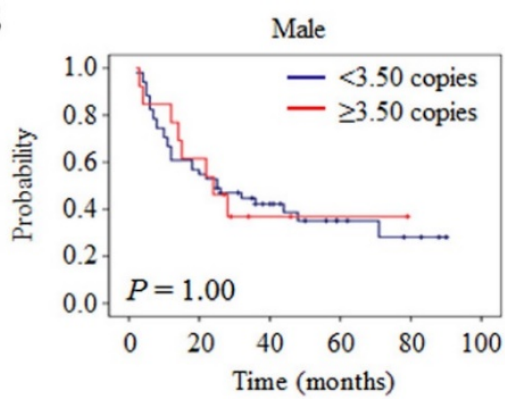

C

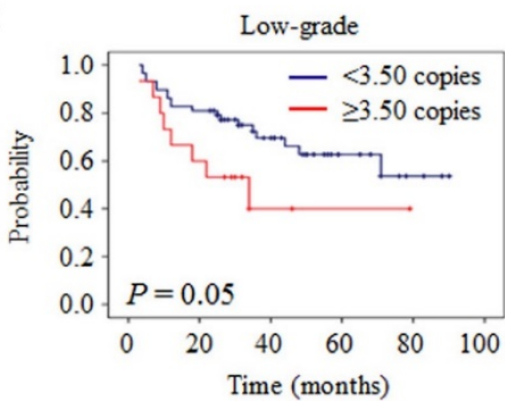

D

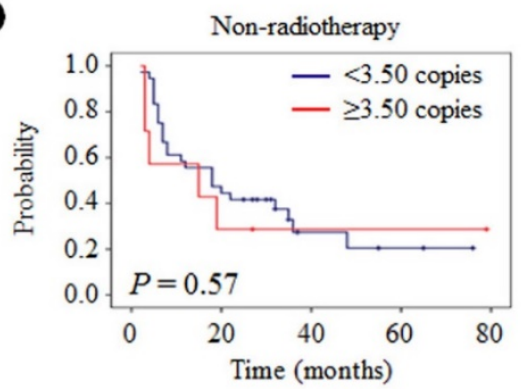

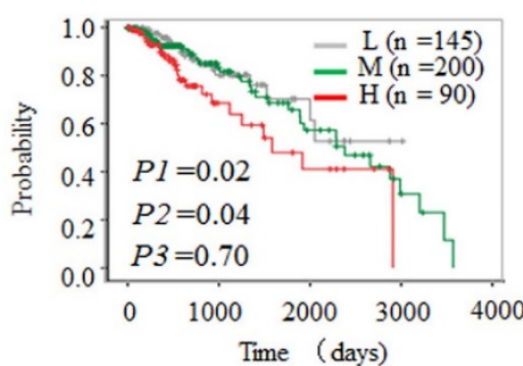
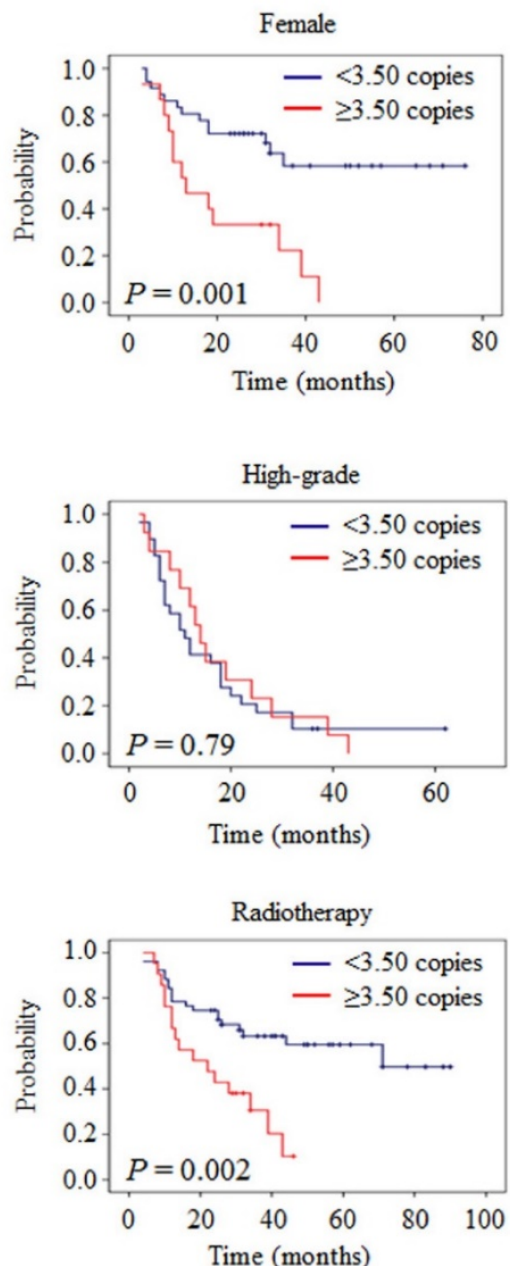

Figure 4. The impact of $A I B I$ amplification on the survival of glioma patients. (A) Kaplan-Meier survival curves were grouped based on the status of $A I B I$ amplification in gliomas from our cohort (left panel) and TCGA cohort (right panel). The presence of AIBI amplification caused a poorer overall survival than the absence of $A I B I$ amplification in female patients (B), the patients with low-grade tumors (C), and the patients receiving radiotherapy (D), but not in male patients, the patients with high-grade tumors and the patients who did not receive radiotherapy. L, low copy number of $A I B I$ (L-group); $M$, medium copy number of $A I B I$ (M-group); H, high copy number of $A I B I$ (H-group); PI for H-group vs. L-group; P2 for H-group vs. M-group; P3 for M-group vs. L-group.

Next, we used the Kaplan-Meier survival analysis to confirm the impact of AIB1 amplification on patient survival. As expected, our data showed a significantly poorer survival in the patients with AIB1 amplification than those without amplification (18.0 months vs. 36.0 months; $P=0.03$ ) (Table 7 and Figure 4A, left panel). This was supported by the TCGA dataset that high copy number of AIB1 was closely related with worse survival as compared with medium $(P=0.04)$ and low copy number $(P=0.02)$, respectively (Figure $4 \mathrm{~A}$, right panel). In addition, to exclude the effect of chemotherapy and radiotherapy on patient survival, we only evaluated the prognostic value of AIB1 amplification in the patients who did not receive any therapy. As expected, we still found that AIB1 amplification was significantly related to poor survival of these patients (Supplementary Figure 1). Further analysis revealed that AIB1 amplification 
almost did not influence the survival of male patients (Figure 4B, left panel). However, it significantly shortened the survival times in female patients (Figure 4B, right panel). Accordingly, 1-, 2- and 3-year overall survival rates were much worse in female patients than male patients (Table 7). When the data were stratified according to WHO grade, we found that AIB1 amplification was related to poor survival in patients with low-grade gliomas, but not in high-grade gliomas, although no statistical significance was obtained (Table 7 and Figure 4C).

\section{Effect of AIB I Amplification on Radiotherapy Outcome of Glioma Patients}

Given that radiotherapy is an important adjuvant therapy after surgical resection for glioma patients, we thus tested the effect of AIB1 amplification on radiotherapy outcome in a cohort of gliomas. As shown in Figure 4D, AIB1 amplification significantly shortened median survival times in the patients receiving radiotherapy (22.0 months vs. 71.0 months; $P=0.002$ ), but not in the patients who did not receive radiotherapy (15.0 months vs. 18.0 months; $P$ $=0.57$ ). Accordingly, AIB1 amplification was markedly associated with worse overall survival rates in the former, but not in the latter (Table 7). In addition, we did not find significant effect of AIB1 amplification on chemotherapy outcome in glioma patients (Supplementary Figure 2). Collectively, our data indicate that AIB1amplification may be considered as a predictor of radiotherapy resistance in gliomas.

\section{Discussion}

Malignant glioma, a common primary tumor of central nervous system, is characterized by complex molecular heterogeneity and associated with poor clinical outcomes of patients [2, 28]. Therefore, understanding the underlying molecular disease mechanisms may lead to better management and appropriate therapeutic strategies to improve clinical outcomes of glioma. Gene amplification is a well-known prevalent mechanism of oncogene overexpression in human cancers [6]. Recent genomic studies in gliomas have shown frequent changes in DNA copy number, resulting in high levels of chromosomal instability $[7,29]$. As a critical oncogene, AIB1 amplification has been widely found in different types of cancer [30]. However, the association of AIB1 amplification with therapeutic outcomes of glioma patients, and prognostic value of AIB1 amplification in gliomas remains totally unknown. In the present study, we compared the copy number of AIB1gene between glioma patients and control subjects, and determined the prognostic significance of AIB1amplification in gliomas. Our data showed that AIB1 was frequently amplified in gliomas, but not in control subjects. Moreover, our data showed that female glioma patients had higher AIB1 copy number as compared to male patients. This was supported by a previous study that female patients had higher AIB1 expression than male patients in NSCLC [31]. Another study demonstrated that there was a significantly higher AIB1 expression in high-grade astrocytomas than that in low-grade astrocytomas, and high AIB1 expression was associated with poor prognosis [24]. These results suggest that AIB1 amplification is likely involved in glioma tumorigenesis.

Table 7. Overall survival by grouping with $A I B I$ amplification.

\begin{tabular}{|c|c|c|c|c|c|c|}
\hline \multirow[t]{2}{*}{ Characteristics } & \multirow[t]{2}{*}{$\mathbf{n}$} & \multicolumn{3}{|c|}{ Overall survival rate $(\%)$} & \multicolumn{2}{|c|}{ Overall survival time (months) } \\
\hline & & 1 year $(95 \% \mathrm{CI})$ & 2 years $(95 \% \mathrm{CI})$ & 3 years $(95 \% \mathrm{CI})$ & Median & $95 \% \mathrm{CI}$ \\
\hline \multicolumn{7}{|l|}{ Total patients } \\
\hline$<3.50$ copies & 87 & $69.0(59.2-78.8)$ & $60.9(50.7-71.1)$ & $49.2(38.0-60.4)$ & 36.0 & $19.3-52.7$ \\
\hline$\geq 3.50$ copies & 28 & $64.3(46.5-82.1)$ & $39.3(21.3-57.3)$ & $29.5(11.3-47.7)$ & 18.0 & $7.6-28.4$ \\
\hline \multicolumn{7}{|l|}{ Male } \\
\hline$<3.50$ copies & 51 & $60.8(47.5-74.1)$ & $52.9(39.2-66.6)$ & $42.1(28.2-56.0)$ & 25.0 & $9.3-40.7$ \\
\hline$\geq 3.50$ copies & 13 & $76.9(54.0-99.8)$ & $46.2(19.2-73.2)$ & $36.9(9.9-63.9)$ & 24.0 & $9.7-38.3$ \\
\hline \multicolumn{7}{|l|}{ Female } \\
\hline$<3.50$ copies & 36 & $80.6(67.7-93.5)$ & $72.2(57.5-86.9)$ & $58.4(39.8-77.0)$ & Not reached & -- \\
\hline$\geq 3.50$ copies & 15 & $53.3(28.0-78.6)$ & $33.3(9.4-57.2)$ & $22.2(-1.7-46.1)$ & 13.0 & $2.9-23.1$ \\
\hline \multicolumn{7}{|l|}{ Low-grade } \\
\hline$<3.50$ copies & 58 & $82.8(73.0-92.6)$ & $81.0(71.0-91.0)$ & $69.7(57.0-82.4)$ & Not reached & -- \\
\hline$\geq 3.50$ copies & 15 & $66.7(42.8-90.6)$ & $53.3(28.0-78.6)$ & $40.0(10.4-69.6)$ & 34.0 & $10.4-57.6$ \\
\hline \multicolumn{7}{|l|}{ High-grade } \\
\hline$<3.50$ copies & 29 & $41.4(23.6-59.2)$ & $20.7(6.0-35.4)$ & $10.3(-0.9-21.5)$ & 11.0 & $6.8-15.2$ \\
\hline$\geq 3.50$ copies & 13 & $61.5(35.0-88.0)$ & $23.1(0.2-46.0)$ & $15.4(-4.2-35)$ & 14.0 & $10.5-17.5$ \\
\hline \multicolumn{7}{|c|}{ Non-radiotherapy } \\
\hline$<3.50$ copies & 36 & $55.6(39.3-71.9)$ & $41.7(25.6-57.8)$ & $27.3(10.2-44.4)$ & 18.0 & $6.3-29.7$ \\
\hline$\geq 3.50$ copies & 7 & $57.1(20.4-93.8)$ & $38.6(6.3-62.1)$ & $28.6(-4.9-62.1)$ & 15.0 & $0.0-43.2$ \\
\hline \multicolumn{7}{|l|}{ Radiotherapy } \\
\hline$<3.50$ copies & 51 & $78.4(67.0-89.8)$ & $74.5(62.5-86.5)$ & $63.2(49.5-76.9)$ & 71.0 & -- \\
\hline$\geq 3.50$ copies & 21 & $66.7(46.5-86.9)$ & $42.9(21.7-64.1)$ & $30.5(9.1-51.9)$ & 22.0 & $7.0-37.0$ \\
\hline
\end{tabular}


Next, we investigated the clinical significance and prognostic value of AIB1 amplification in a cohort of gliomas. The results demonstrated that AIB1 amplification was significantly correlated with WHO grade and tumor recurrence, and was an independent risk factor for cancer-related death in female glioma patients. Given the association of high AIB1 expression with poor prognosis of glioma patients [24], we investigated the impact of AIB1 amplification on patient survival. As expected, AIB1 amplification was closely related to poor patient survival, particularly in female patients. Multivariate analysis demonstrated that AIB1 amplification was an independent risk factor for poor patient survival. These findings suggest that this molecular event may contribute to clinical outcomes, and may thus serve as a potential therapeutic target in glioma patients, particularly in female patients.

AIB1as a steroid receptor coactivator of estrogen and progesterone receptors can enhance the transcription of target genes through being recruited to their promoters or enhancers via nuclear receptors $[12,32,33]$. In addition, there is evidence showing that estradiol can enhance the transcriptional activity of AIB1 through increasing its phosphorylation and decreasing its sumoylation [34]. Estradiol also promotes the proliferation of astrocytoma cells through estrogen receptor- $\alpha$ (ERa) and its interaction with AIB1 [35]. It is the fact that AIB1 interacts with $E R a$, and subsequently binds to ERa-binding site on the promoter of SNAI1, a transcriptional repressor for E-cadherin, to promote the transcription of SNAI1and repress E-cadherin expression, ultimately leading to the initiation and progression of epithelial mesenchymal transition (EMT) [36]. Thus, we speculate that specific role and prognostic value of AIB1 amplification in female glioma patients may be related to sex hormones and nuclear receptor levels.

Further analysis revealed that AIB1 amplification was only related to poor patient survival in low-grade tumors, but not in high-grade tumors. Moreover, AIB1 copy number did not show a significant difference between them. These findings indicate that AIB1 amplification may be an early molecular event, and may affect the prognosis in early stage of glioma tumorigenesis. Interestingly, we observed that AIB1 amplification significantly impacted radiotherapy outcome in glioma patients. Similar to these findings, a previous study showed that AIB1 was related to chemo-radiotherapy (CRT) response in esophageal squamous cell carcinoma (ESCC). Compared to the CRT-effective group, AIB1 overexpression was more frequently found in the CRT-resistant group [37]. However, the underlying molecular mechanism is still unknown. The PI3K/AKT/mTOR signal pathway has been identified as the predominant downstream pathway of AIB1 [38, 39], contributing to radioresistance in different types of cancer including glioma [40-47]. Thus, we speculate that the activation of PI3K/AKT pathway may be one of molecular mechanisms underlying AIB1-induced radiotherapy resistance.

In summary, we found frequent AIB1 amplification in gliomas, and demonstrated that this genetic event was closely related to poor prognosis in glioma patients, particularly in female patients. To our knowledge, our data for the first time reveals that AIB1 amplification affects radiotherapy outcome in glioma patients. Collectively, these observations raise the possibility that AIB1 amplification may be one of major driving forces in glioma tumorigenesis, and may be potentially prognostic marker for glioma patients particularly female patients.

\section{Abbreviations}

AA: anaplastic astrocytoma; AIB1: Amplified in breast cancer 1; CI: confidence interval; CNAs: copy number alterations; CNS: central nervous system; CRT: chemo-radiotherapy; DA: diffuse astrocytoma; DNA: deoxyribonucleic acid; EMT: epithelial mesenchymal transition; ERa: estrogen receptor alpha; ESCC: esophageal squamous cell carcinoma; FISH: fluorescence in situ hybridization; GBM: glioblastoma; H\&E: hematoxylin and eosin; HR: hazard ratio; KPS: Karnofsky performance status; mRNA: messenger ribonucleic acid; NSCLC: non small cell lung cancer; OR: odds ratio; PA: pilocytic astrocytoma; PCR: polymerase chain reaction; SRC-3: steroid receptor coactivator-3; SDS: sodium dodecylsulfate; TCGA: The Cancer Genome Atlas; WHO: World Health Organization.

\section{Supplementary Material}

Supplementary figures.

http://www.jcancer.org/v07p2052s1.pdf

\section{Acknowledgments}

This work was supported by Key Science and Technology Program of Shaanxi Province (No. 22015SF036), and the Fundamental Research Funds for the Central Universities.

\section{Competing Interests}

The authors have declared that no competing interest exists. 


\section{References}

1. Dolecek TA, Propp JM, Stroup NE, et al. CBTRUS statistical report: primary brain and central nervous system tumors diagnosed in the United States in 2005-2009. Neuro Oncol. 2012; 14 (Suppl 5): v1-49.

2. Ducray F, Idbaih A, Wang XW, et al. Predictive and prognostic factors for gliomas. Expert Rev Anticancer Ther. 2011; 11: 781-9.

3. Louis DN, Ohgaki H, Wiestler OD, et al. The 2007 WHO classification of tumours of the central nervous system. Acta Neuropathol. 2007; 114: 97-109.

4. Catuogno S, Esposito CL, Quintavalle C, et al. Nucleic acids in human glioma treatment: innovative approaches and recent results. J Signal Transduct. 2012; 2012: 735135 .

5. Ohgaki H. Epidemiology of brain tumors. Methods Mol Biol. 2009; 472: 323-42.

6. Schwab M. Amplification of oncogenes in human cancer cells. Bioessays. 1998; 20: 473-9.

7. Cancer Genome Atlas Research Network. Comprehensive genomic characterization defines human glioblastoma genes and core pathways. Nature. 2008; 455: 1061-8.

8. Rao SK, Edwards J, Joshi AD, et al. A survey of glioblastoma genomic amplifications and deletions. J Neurooncol. 2010; 96: 169-79.

9. Burton EC, Lamborn KR, Feuerstein BG, et al. Genetic aberrations defined by comparative genomic hybridization distinguish long-term from typical survivors of glioblastoma. Cancer Res. 2002; 62: 6205-10.

10. Inda MM, Fan X, Munoz J, et al. Chromosomal abnormalities in human glioblastomas: gain in chromosome $7 \mathrm{p}$ correlating with loss in chromosome 10q. Mol Carcinog. 2003; 36: 6-14.

11. Suen CS, Berrodin TJ, Mastroeni R, et al. A transcriptional coactivator, steroid receptor coactivator-3, selectively augments steroid receptor transcriptional activity. J Biol Chem. 1998; 273: 27645-53.

12. Anzick SL, Kononen J, Walker RL, et al. AIB1, a steroid receptor coactivator amplified in breast and ovarian cancer. Science. 1997; 277: 965-8.

13. Xu FP, Xie D, Wen JM, et al. SRC-3/AIB1 protein and gene amplification levels in human esophageal squamous cell carcinomas. Cancer Lett. 2007; 245: 69-74.

14. Xie D, Sham JS, Zeng WF, et al. Correlation of AIB1 overexpression with advanced clinical stage of human colorectal carcinoma. Hum Pathol. 2005; 36: 777-83.

15. Wang Y, Wu MC, Sham JS, et al. Prognostic significance of c-myc and AIB1 amplification in hepatocellular carcinoma. A broad survey using high-throughput tissue microarray. Cancer. 2002; 95: 2346-52.

16. Sakakura C, Hagiwara A, Yasuoka R, et al. Amplification and over-expression of the AIB1 nuclear receptor co-activator gene in primary gastric cancers. Int J Cancer. 2000; 89: 217-23.

17. Ghadimi BM, Schrock E, Walker RL, et al. Specific chromosomal aberrations and amplification of the AIB1 nuclear receptor coactivator gene in pancreatic carcinomas. Am J Pathol. 1999; 154: 525-36.

18. Luo JH, Xie D, Liu MZ, et al. Protein expression and amplification of AIB1 in human urothelial carcinoma of the bladder and overexpression of AIB1 is a new independent prognostic marker of patient survival. Int J Cancer. 2008; 122: 2554-61.

19. Liu MZ, Xie D, Mai SJ, et al. Overexpression of AIB1 in nasopharyngeal carcinomas correlates closely with advanced tumor stage. Am J Clin Pathol. 2008; 129: 728-34.

20. He LR, Zhao HY, Li BK, et al. Overexpression of AIB1 negatively affects survival of surgically resected non-small-cell lung cancer patients. Ann Oncol. 2010; 21: 1675-81.

21. Tabach Y, Kogan-Sakin I, Buganim Y, et al. Amplification of the 20q chromosomal arm occurs early in tumorigenic transformation and may initiate cancer. PLoS One. 2011; 6: e14632.

22. Carvalho B, Postma C, Mongera S, et al. Multiple putative oncogenes at the chromosome $20 \mathrm{q}$ amplicon contribute to colorectal adenoma to carcinoma progression. Gut. 2009; 58: 79-89.

23. Wullich B, Riedinger S, Brinck $U$, et al. Evidence for gains at $15 \mathrm{q}$ and $20 \mathrm{q}$ in brain metastases of prostate cancer. Cancer Genet Cytogenet. 2004; 154: 119-23.

24. Kefalopoulou $\mathrm{Z}$, Tzelepi $\mathrm{V}$, Zolota $\mathrm{V}$, et al Prognostic value of novel biomarkers in astrocytic brain tumors: nuclear receptor co-regulators AIB1, TIF2, and PELP1 are associated with high tumor grade and worse patient prognosis. J Neurooncol. 2012; 106: 23-31.

25. Ji M, Guan H, Gao C, et al. Highly frequent promoter methylation and PIK3CA amplification in non-small cell lung cancer (NSCLC). BMC Cancer. 2011; $11: 147$.

26. Wu G, Mambo E, Guo $Z$, et al. Uncommon mutation, but common amplifications, of the PIK3CA gene in thyroid tumors. J Clin Endocrinol Metab. 2005; 90: 4688-93

27. Engelman JA, Zejnullahu K, Mitsudomi T, et al. MET amplification leads to gefitinib resistance in lung cancer by activating ERBB3 signaling. Science. 2007; 316: 1039-43.

28. Assem M, Sibenaller Z, Agarwal S, et al. Enhancing diagnosis, prognosis, and therapeutic outcome prediction of gliomas using genomics. OMICS. 2012; 16: 113-22.

29. Parsons DW, Jones $S$, Zhang $X$, et al. An integrated genomic analysis of human glioblastoma multiforme. Science 2008; 321: 1807-12.

30. $\mathrm{Xu} \mathrm{J}, \mathrm{Wu} \mathrm{RC}, \mathrm{O}^{\prime}$ Malley BW. Normal and cancer-related functions of the p160 steroid receptor co-activator (SRC) family. Nat Rev Cancer. 2009; 9: 615-30.
31. Wang $\mathrm{H}$, Zhang $\mathrm{D}, \mathrm{Wu} \mathrm{W}$, et al. Overexpression and gender-specific differences of SRC-3 (SRC-3/AIB1) immunoreactivity in human non-small cell lung cancer: an in vivo study. J Histochem Cytochem. 2010; 58: 1121-7.

32. Han SJ, DeMayo FJ, Xu J, et al. Steroid receptor coactivator (SRC)-1 and SRC-3 differentially modulate tissue-specific activation functions of the progesterone receptor. Mol Endocrinol. 2006; 20: 45-55.

33. McKenna NJ, O'Malley BW. Combinatorial control of gene expression by nuclear receptors and coregulators. Cell. 2002; 108: 465-74.

34. Wu H, Sun L, Zhang Y, et al. Coordinated regulation of AIB1 transcriptional activity by sumoylation and phosphorylation. J Biol Chem. 2006; 281: 21848-56.

35. Gonzalez-Arenas A, Hansberg-Pastor V, Hernandez-Hernandez OT, et al Estradiol increases cell growth in human astrocytoma cell lines through ERalpha activation and its interaction with SRC-1 and SRC-3 coactivators. Biochim Biophys Acta. 2012; 1823: 379-86.

36. Wang M, Zhao F, Li S, et al. AIB1 cooperates with ERalpha to promote epithelial mesenchymal transition in breast cancer through SNAI1 activation. PLoS One. 2013; 8: e65556.

37. He LR, Liu MZ, Li BK, et al. Overexpression of AIB1 predicts resistance to chemoradiotherapy and poor prognosis in patients with primary esophageal squamous cell carcinoma. Cancer Sci. 2009; 100: 1591-6.

38. Torres-Arzayus MI, Font de Mora J, Yuan J, et al. High tumor incidence and activation of the PI3K/AKT pathway in transgenic mice define AIB1 as an oncogene. Cancer Cell. 2004; 6: 263-74.

39. Torres-Arzayus MI, Yuan J, DellaGatta JL, et al. Targeting the AIB1 oncogene through mammalian target of rapamycin inhibition in the mammary gland. Cancer Res. 2006; 66: 11381-8.

40. Ni J, Cozzi P, Hao J, et al. Epithelial cell adhesion molecule (EpCAM) is associated with prostate cancer metastasis and chemo/radioresistance via the PI3K/Akt/mTOR signaling pathway. Int J Biochem Cell Biol. 2013; 45: 2736-48.

41. Chang L, Graham PH, Hao I, et al. Acquisition of epithelial-mesenchymal transition and cancer stem cell phenotypes is associated with activation of the $\mathrm{PI} 3 \mathrm{~K} / \mathrm{Akt} / \mathrm{mTOR}$ pathway in prostate cancer radioresistance. Cell Death Dis. 2013; 4: e875.

42. Gupta AK, McKenna WG, Weber CN, et al. Local recurrence in head and neck cancer: relationship to radiation resistance and signal transduction. Clin Cancer Res. 2002; 8: 885-92.

43. Blackhall $\mathrm{FH}$, Pintilie $\mathrm{M}$, Michael $\mathrm{M}$, et al. Expression and prognostic significance of kit, protein kinase $\mathrm{B}$, and mitogen-activated protein kinase in patients with small cell lung cancer. Clin Cancer Res. 2003; 9: 2241-7.

44. Choe G, Horvath S, Cloughesy TF, et al. Analysis of the phosphatidylinositol 3 '-kinase signaling pathway in glioblastoma patients in vivo. Cancer Res. 2003; 63: $2742-6$

45. Liao Y, Grobholz R, Abel U, et al. Increase of AKT/PKB expression correlates with gleason pattern in human prostate cancer. Int J Cancer. 2003; 107: 676-80.

46. Schmitz KJ, Otterbach F, Callies R, et al. Prognostic relevance of activated Akt kinase in node-negative breast cancer: a clinicopathological study of 99 cases. Mod Pathol. 2004; 17: 15-21.

47. Yamamoto S, Tomita Y, Hoshida $Y$, et al. Prognostic significance of activated Akt expression in pancreatic ductal adenocarcinoma. Clin Cancer Res. 2004; 10: $2846-50$. 